\title{
Klippel-Feil Syndrome
}

National Institute of Neurological Disorders and Stroke (NINDS)

\section{Source}

National Institute of Neurological Disorders and Stroke (NINDS). Klippel-Feil Syndrome

Information Page.

Klippel-Feil Syndrome is a rare disorder characterized by the congenital fusion of two or more cervical (neck) vertebrae. It is caused by a failure in the normal segmentation or division of the cervical vertebrae during the early weeks of fetal development. The most common signs of the disorder are short neck, low hairline at the back of the head, and restricted mobility of the upper spine. The fused vertebrae can cause nerve damage and pain in the head, neck, or back. Associated abnormalities may include scoliosis (curvature of the spine), spina bifida (a birth defect of the spine), cleft palate, respiratory problems, and heart malformations. Other features may include joint pain; anomalies of the head and face, skeleton, sex organs, muscles, brain and spinal cord, arms, legs, and fingers; and difficulties hearing. Most cases are sporadic (happen on their own) but mutations in the GDF6 (growth differentiation factor 6) or GDF3 (growth differentiation factor 3) genes can cause the disorder. These genes make proteins that are involved in bone development and segmentation of the vertebrae. 